\title{
MEKK3 Sustains EMT and Stemness in Pancreatic Cancer by Regulating YAP and TAZ Transcriptional Activity
}

\author{
RAFFAELA SANTORO ${ }^{1 *}$, MARCO ZANOTTO ${ }^{1 *}$, CARMINE CARBONE ${ }^{1}$, \\ GENY PIRO $^{1,2}$, GIAMPAOLO TORTORA ${ }^{2,3}$ and DAVIDE MELISI ${ }^{1,3}$ \\ ${ }^{1}$ Digestive Molecular Clinical Oncology Research Unit, Department of Medicine, \\ Università degli studi di Verona, Verona, Italy; \\ ${ }^{2}$ Laboratory of Oncology and Molecular Therapy, Department of Medicine, \\ Università degli studi di Verona, Verona, Italy; \\ ${ }^{3}$ Medical Oncology Unit, Azienda Ospedaliera Universitaria Integrata, Verona, Italy
}

\begin{abstract}
Background/Aim: Pancreatic cancer is one of the most threatening and poorly understood human malignancies. MEKK3 (MAP3K3) is a serinelthreonine kinase activated by different signaling pathways. YAP and TAZ are critical oncogenic effectors in pancreatic cancer. We hypothesized that MEKK3 could sustain pancreatic cancer by inducing YAP/TAZ oncogenic activities. Materials and Methods: In Panc1 and AsPC1 pancreatic cancer cell lines MEKK3 was knocked-out (KO) by the CRISPR/Cas9 method. These cells were used to evaluate MEKK3 contribution to the expression of YAP/TAZ and their target genes, cell migration, stemness, and in vivo tumor growth. Results: MEKK3 KO reduced both $E M T$ and cell migration, the size of $3 D$ colonies and the percentage of $C D 44^{+} / C D 24^{+} / E p C A M^{+} C S C$, promoter recruitment of YAP/TAZ and the expression of their target genes. It reduced tumor growth and prolonged mice overall survival. Conclusion: Silencing of MEKK3 represents a valid approach to revert in vivo the aggressiveness of pancreatic cancer by modulating YAP/TAZ transcriptional activities.
\end{abstract}

Pancreatic cancer remains one of the most lethal and poorly understood human malignancies $(1,2)$. It has the lowest 5 -year relative survival rate among solid tumors at $8 \%$ (3), and is projected to become the second leading cause of

This article is freely accessible online.

*These Authors contributed equally to this work.

Correspondence to: Dr. Davide Melisi, Digestive Molecular Clinical Oncology unit, Section of Medical Oncology, Department of Medicine, University of Verona, AOUI Verona, Policlinico "G.B. Rossi", Piazzale L.A. Scuro, 10, 37134, Verona, Italy. Tel: +39 045 8128148 e-mail: davide.melisi@univr.it

Key Words: MEKK3, YAP, TAZ, pancreatic cancer. cancer-related death by 2030 in Western countries (4). Poor prognosis in pancreatic cancer is attributed to its early metastatic behavior, aggressive clinical course, and limited efficacy of chemotherapeutic treatments $(5,6)$.

Epithelial-to-mesenchymal transition (EMT) is a wellcoordinated process triggered by many signaling pathways during embryonic development. However, its aberrant activation in cancer development has been associated with cancer stem cell properties, self-renewal capabilities, resistance to conventional therapies, and endow cancer cells with the migratory and invasive capabilities associated with metastatic competence (7).

In the last decade, we contributed to this field by demonstrating that different paracrine inflammatory signals - including Interleukin-1 (IL-1) (8) - could induce EMT and, in turn, metastasis and treatment resistance in colorectal (9, 10) and pancreatic cancer (11). During the immune and inflammatory responses, the IL-1- induced TRAF-6 signaling leads to the activation of NF-KB (12) - a key transcriptional factor that orchestrates expression of many genes involved in inflammation, oncogenesis, and apoptosis $(13,14)-$ through two parallel signaling pathways depending upon the activation of two Mitogen-activated kinase kinase kinases (MAP3Ks), the TGF- $\beta$-activated kinase 1 (TAK1; MAP3K7) $(15,16)$, and the MAP3K3, or MEKK3 $(17,18)$.

MEKK3 is a serine/threonine kinase belonging to the MEKK/STE11 subgroup of the MAP3K family that is constitutively expressed in several types of tissues. A pivotal role for MEKK3 has been demonstrated in orchestrating cellular processes such as proliferation, cell cycle progression, differentiation, migration, apoptosis and inflammatory response (17, 19), through the integration of different signaling pathways, such as TNF $\alpha$ (20), IL-1 (17) and TLR4 (21).

The transcriptional regulators yes-associated protein (YAP) and transcriptional coactivator with PDZ-binding motif (TAZ) are emerging as key players in cancer initiation and progression. 
In particular, by forming complexes with transcription factors such as TEA domain family members (TEADs), AP1, SMADs and nucleosome remodeling deacetylase (NuRD) complex, they drive the transcription of genes involved in cell cycle progression, cell proliferation, differentiation, angiogenesis, stress response, apoptosis, and extracellular matrix formation (22-24), as well as in maintaining self-renewal and tumorinitiation capacity of Cancer Stem Cells (CSC) $(22,25)$.

In the present study, we hypothesized that MEKK3 could mediate EMT and stemness in pancreatic cancer by regulating YAP and TAZ oncogenic activities independently of its effect on NF-kB transcriptional activity.

\section{Materials and Methods}

Cell lines and reagents. Human pancreatic cancer cell lines AsPC1 and Panc1 were purchased from the American Type Culture Collection (Manassas, VA, USA) and cultured as in (15).

Generation of knock-out cell lines. Panc1 and AsPC1 cells were transfected with plasmids expressing either control or MEKK3 targeting guide RNAs (Table I), as well as with Cas9-RFP vectors (Transomic, Huntsville, AL, USA) using OMNIfect transfection reagent (\#OTR1001, Transomic) following manufacturer's instruction and selected with $2 \mu \mathrm{g} / \mathrm{ml}$ Blasticidin (A1113902, Life Technologies, Carlsbad, CA, USA) for $72 \mathrm{~h}$. RFP-positive cells were single-sorted (FACS CANTO ARIA II, BD, Franklin Lakes, NJ, USA) into 96-well plates. Single clones were expanded and screened for MEKK3 expression by protein immunoblotting.

Quantitative real-time PCR ( $q R T$-PCR). qRT-PCR was performed as previously described in (26). Primers for IL1 $\alpha$ (QT00001127), IL1 $\beta \quad$ (QT00021385), CXCL1 (QT00199752), CXCL2 (QT00013104), CXCL3 (QT00015442) and IL8 (QT00000322) were purchased from QIAGEN (Hilden, Germany). All the other primers used for amplification are listed in Table II.

Western blotting analyses. Western blot analyses were performed as described in (27). Anti-CDH1 (ab40772, 1:10000) antibody was purchased from Abcam (Cambridge, UK). YAP/TAZ (sc-101199, 1:1,000), CTGF (sc-14939, 1:1000), GAPDH (sc-166545, 1:50,000), as well as secondary HRP-conjugated anti-mouse (sc-2318) and antirabbit (sc-2030) antibodies were purchased from Santa Cruz Biotechnology (Santa Cruz, CA, USA). MEKK3 (\#5727, 1:1,000), AXL (\#3269, 1:1000), p65 (\#4764, 1:1,000), p-p65 (\#3031, 1:1,000) were purchased by Cell Signaling (Danvers, MA, USA) and vimentin (M0725, 1:4,000) was purchased DakoCytomation (Glostrup, Denmark). Images were acquired using ImageQuant LAS 4000 mini (GE Healthcare Life Sciences, Little Chalfont, UK).

Migration assays. Transwell migration assays were performed as described in (28) and wound healing (WH) assay as in (29). For transwell assays, cells were automatically counted using ImageJ software. For WH, photographs of at least three different points were taken every hour for $72 \mathrm{~h}$. Images were acquired by fluorescence microscopy (EVOS FL Auto, Thermo Fisher Scientific, Carlsbad, CA, USA). Wound opening was measured using the Image J WH tool plugin.
Table I. Sequences of control and MEKK3 targeting guide RNA.

\begin{tabular}{lcl}
\hline Gene & Clone ID & Sequence \\
\hline MAP3K3 & TEVH-1081756 & GGACATTCGTGATTTCCGGA \\
MAP3K3 & TEVH-1148898 & CCTTGTGGTGCACAGACACG \\
MAP3K3 & TEVH-1216040 & ACAGACACGTGGTAGCGCCG \\
TELG1012 & Non targeting control & GGAGCGCACCATCTTCTTCA \\
\hline
\end{tabular}

Cell proliferation assays. $1.0 \times 10^{3}$ MEKK3 KO or control cells were seeded in 96 -well plates. Cell proliferation was measured by using the Sulforhodamine B (SRB) assay, as in (30).

Colony-forming assay. Colony-forming assay was performed as previously described in (31). Colonies were counted by automatic microscopy (EVOS FL Auto).

Chromatin immunoprecipitation (ChIP) assay. Cells were crosslinked and chromatin was extracted and sonicated. Chromatin was incubated overnight with the following antibodies: IgG (Vector Laboratories, X0720, 1:50, Burlingame, CA, USA), anti-PhosphoNF-kB (\#3033, 1:50 Cell Signaling), anti-YAP (Santa Cruz Biotechnology, sc-101199, $1 \mu \mathrm{g}$, Santa Cruz, CA, USA). Dynabeads Protein G were blocked overnight with $1 \mathrm{mg} / \mathrm{ml}$ Sonicated Salmon Sperm (Thermo Fisher Scientific, 15632011, Carlsbad, CA, USA) and $1 \mathrm{mg} / \mathrm{ml}$ Bovine Serum Albumin (Thermo Fisher Scientific, AM2616, Carlsbad, CA, USA). Thirty $\mu$ l Dynabeads Protein G (50\% slurry) were used for each IP. Immunoprecipitated (IPed) chromatin was purified with DNeasy Blood \& Tissue Kit (Qiagen, 69504, Hilden, Germany) following manufacturer's instruction. DNA enrichment was assessed by qRT-PCR (primers listed in Table III). IPed chromatin was normalized to input chromatin (GAPDH gene). Histograms show the ratio of Ab-specific IPed chromatin normalized to $\mathrm{IgG}$.

Nude mouse orthotopic xenograft models. Five-weeks old female athymic nude mice (Crl:CD1-Foxn1nu, CDNSSFE05S) were purchased from Charles River (Wilmington, MA, USA). All mice were housed and treated in accordance with the guidelines of the University of Verona Animal Ethic Committee, and maintained in specific pathogen-free conditions. To produce pancreatic tumors, $1.0 \times 10^{4} \mathrm{cells} / \mu \mathrm{l}$ in a solution of 1:1 Matrigel:PBS (Matrigel Matrix Growth Factor Reduced, 356230, BD, Franklin Lakes, NJ) were orthotopically injected as described in (32). Bulky disease was considered present when the tumor burden was prominent in the mouse abdomen (tumor volume $\geq 2,000 \mathrm{~mm}^{3}$ ). The median survival duration was considered reached when at least three of five mice in a group presented with bulky disease, and tumor growth was monitored by fluorescence imaging (IVIS 100 imaging system, Xenogen, PerkinElmer, Waltham, MA, USA). Mice were euthanized by cervical dislocation when evidence of advanced bulky disease was present, which was considered the day of death for survival evaluation.

Immunohistochemistry. Formalin-fixed paraffin-embedded tissue sections were subjected to immunostaining using the streptavidinperoxidase technique, with diaminobenzidine (Immpact DAB, 
Table II. Sequences of primers used in qRT-PCR.

\begin{tabular}{lll}
\hline Primer & FW Sequence & Rev Sequence \\
\hline CTGF & TACCAATGACAACGCCTCCT & TGCCCTTCTTAATGTTCTCTTCC \\
DKK1 & AAAAATGTATCACACCAAAGGACAAG & ATCCTGAGGCACAGTCTGATGA \\
BDNF & AGTTCGGCCCAATGAAGAAA & GAGCATCACCCTGGACGTGTA \\
FOSL1 & GCAGGCGGAGACTGACAAAC & TTCCGGGATTTTGCAGATG \\
CYR61 & GGATCTGCAGAGCTCAGTCAGA & CTTTCCCCGTTTGGTAGATTCT \\
AXL & TGCGCCAGGGAAATCG & AGGCATACAGTCCATCCAGACA \\
YAP & CCACAGGCAATGCGGAATAT & CTGGCTACGCAGGGCTAACT \\
TAZ & GGTGCTACAGTGTCCCCACAA & TTTCTCCTGTATCCATCTCATCCA \\
BIRC3 & GACAGGAGTTCATCCGTCAAGTT & TCTGATGTGGATAGCAGCTGTTC \\
BAX & TGGAGCTGCAGAGGATGATTG & GCTGCCACTCGGAAAAAGAC \\
BCL2 & GGCTGGGATGCCTTTGTG & CAGCCAGGAGAAATCAAACAGA \\
b-actin & GGCATGGGTCAGAAGGATT & CACACGCAGCTCATTGTAGAAG
\end{tabular}

Table III. Sequences of ChIP primers used in qRT-PCR.

\begin{tabular}{lll}
\hline Primer & FW Sequence & Rev Sequence \\
\hline Bcl 2 & TGCACGTCCCTAGACATTGCT & CGCTGCCCTGCTGTGAA \\
Axl & GAGTGGAGTTCTGGAGGAATGTTT & GTGAGGCCGTGTCTCTCTATCC \\
DKK1 & GAAGGTGCTTAACGCATTGATTC & CCCATCCCAAATCCATCTG \\
FosL1 & GCCTGTGTTCCCAAGGAAAG & CCTTCCATTTCATCAGCTCTAGCT \\
CTGF & GGATCAATCCGGTGTGAGTTG & CCTACACAAACAGGGACATTCCT \\
\hline
\end{tabular}

SK-4105, Vector Lab, Burlingame, CA, USA) as a chromogen, as previously described in (33). For antigen retrieval, the sections were subjected to pressure cooker heating in antigen retrieval buffer (either citrate pH 6.0, ab93678 or Tris-EDTA pH 9.0, ab93684, Abcam, Cambridge, UK). Sections were incubated at $4^{\circ} \mathrm{C}$ overnight with Abcam primary rabbit monoclonal antibodies anti-Ki67 (ab92742, 1:4,000), anti-CTGF (ab6992, 1:600), anti-AXL (ab72069, 1:100), anti-MMP7 (ab207299, 1:4,000) and anti-CXCR4 (ab124824, 1:500). Slides were incubated for $30 \mathrm{~min}$ with ImmPRESS ${ }^{\text {TM }}$ HRP AntiRabbit IgG Peroxidase Polymer Detection Kit (MP-7401-50, Vector Lab, Burlingame, CA, USA), counterstained with Vector ${ }^{\circledR}$ Hematoxylin QS (H-3404, Vector Lab, Burlingame, CA, USA) and mounted using VectaMount ${ }^{\mathrm{TM}}$ Permanent Mounting Medium (H5000, Vector Lab, Burlingame, CA, USA).

\section{Results}

MEKK3 KO reverts EMT in pancreatic cancer cell lines. In order to study the contribution of MEKK3 to the aggressiveness of pancreatic cancer, we used CRISPR-Cas9 method to knock-out the expression of MEKK3 in AsPC1 and Panc1 cell lines. With this approach, we were able to completely suppress the expression of MEKK3 in these cell lines (Figure 1A). To determine the effect of targeting MEKK3 expression on the EMT phenotype in pancreatic cancer, we demonstrated that the expression of the epithelial marker gene E-cadherin was significantly upregulated and that of the mesenchymal marker Vimentin was downregulated in both MEKK3 knock-out (KO) cell lines compared with their respective control cell lines (Figure 1B). Consistently, MEKK3 KO cells had significantly lower migration rates than did their respective control cell lines in transwell (Figure 1C) and wound-healing assays (Figure 1D).

To study the contribution of MEKK3 to pancreatic cancer cells stemness, we evaluated the ability of MEKK3 KO or control cells to form colonies, and to form 3D structures in suspension. Panc1 and AsPC1 MEKK3 KO cells showed a significant reduction in colony size (Figure 1E). Likewise, we observed that spheroids formed by MEKK3 KO cell lines were significantly smaller than those formed by their control counterparts (Figure 1F). We further evaluated the percentage of $\mathrm{CD}_{4} 4^{+} / \mathrm{CD} 24^{+} / \mathrm{EpCAM}^{+}$Cancer Stem Cells (CSC) represented in the spheroids arisen by $\mathrm{Ctrl}$ and MEKK3 KO cell lines, and we observed that they were decreased by at least 40\% in MEKK3 KO spheroids (Figure 1G).

Altogether, these data suggest that MEKK3 contributes to both EMT and stemness of pancreatic cancer cells.

MEKK3 KO modulates YAP/TAZ transcriptional activities. In order to understand the molecular mechanisms underlying the oncogenic activity of MEKK3 in pancreatic cancer, we initially investigated whether MEKK3 KO could impair the expression 
A

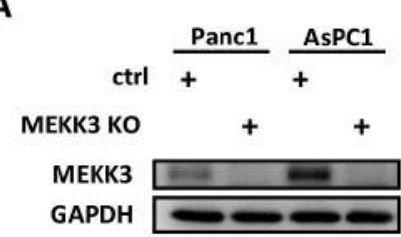

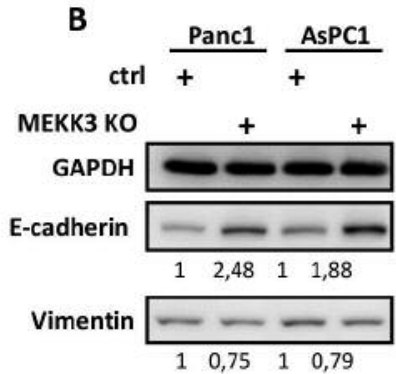
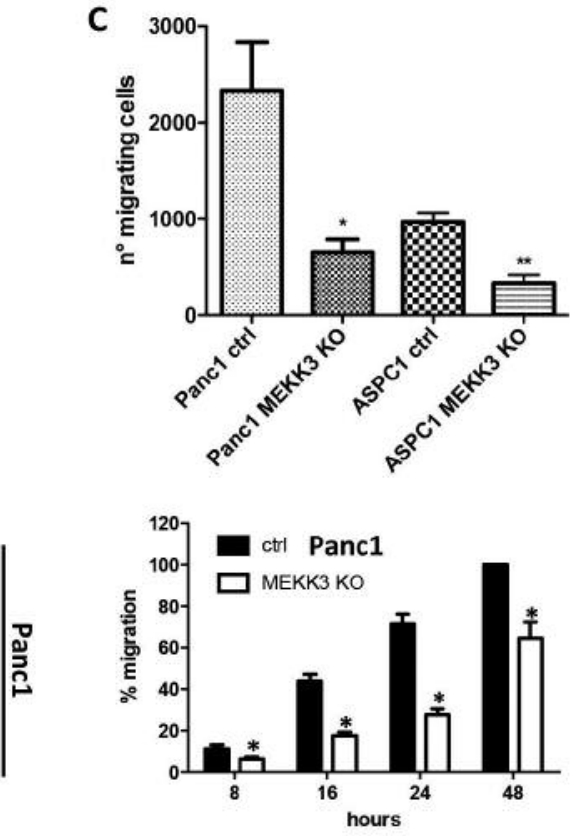
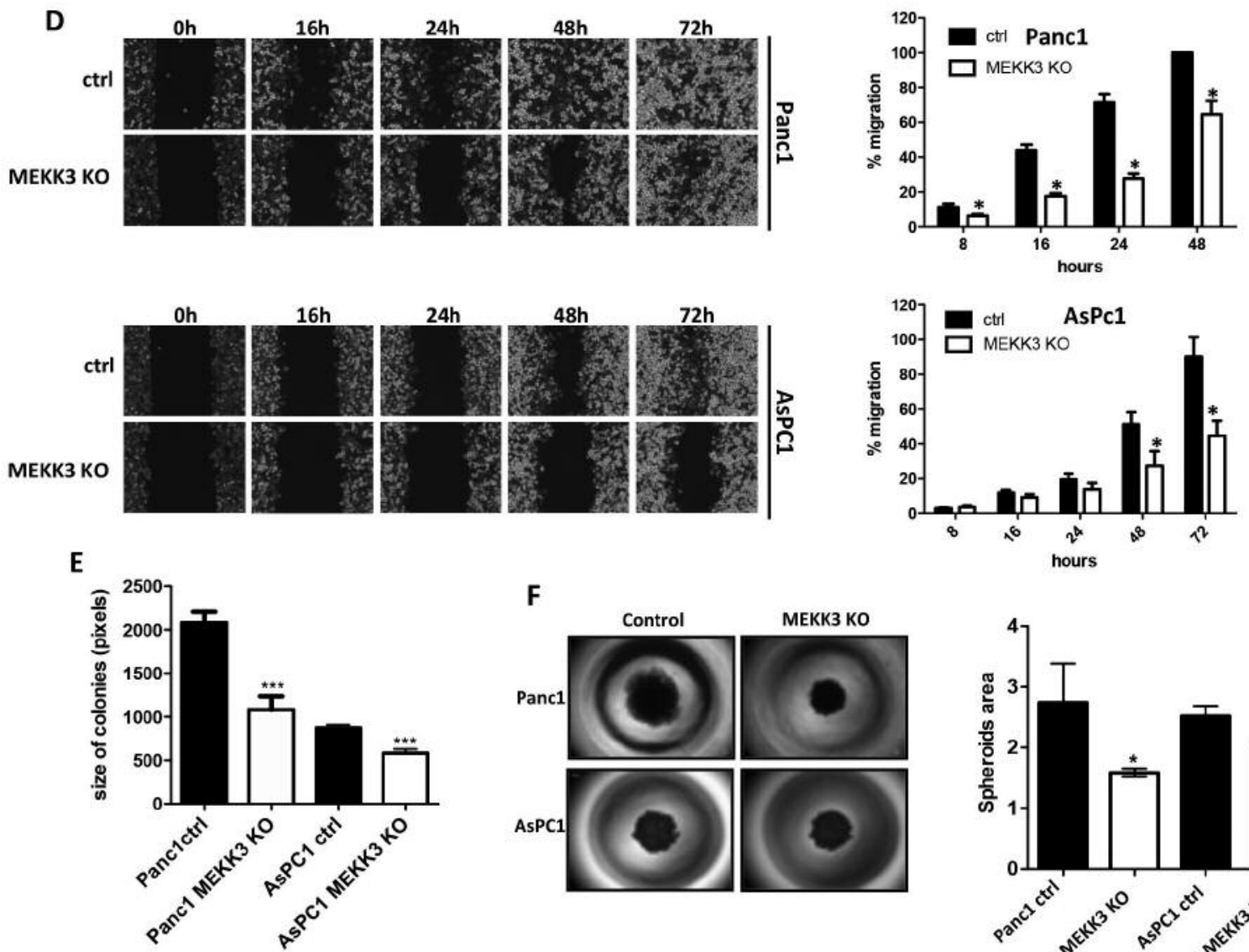

G

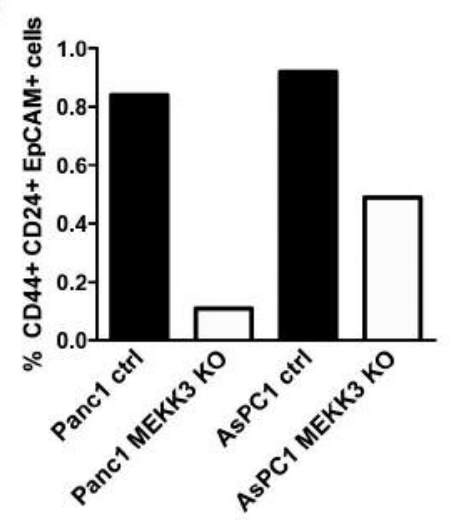

Figure 1. MEKK3 knock-out reverts EMT, proliferation and stemness in Pancl and AsPC1 cell lines. Western blot analysis of the expression of MEKK3 (A) and of EMT markers E-Cadherin and vimentin $(B)$ in whole protein extracts in control and MEKK3 KO Panc1 and AsPC1 cells. C) Transwell migration assays in the indicated control and MEKK3 KO cells; histograms show the number of migrating cells. D) Levels of migration in the indicated cell lines. Results are presented as percentages of the total distances between the wound edges enclosed by cancer cells. Histograms show mean and standard deviations. Representative images at each time point are shown. E) Colony forming assays in Pancl and AsPC1 control and MEKK3 KO cells. Mean and $S D$ are shown in the graphs. ${ }^{* *} p<0.001$ as calculated by the t-test. F) $3 D$ spheroid formation in Pancl and AsPC1 control and MEKK3 KO cells. Histograms show spheroid area. G) Histogram shows the percentage of $C D 44^{+} C D 24^{+} E p C A M+$ cancer stem cells in spheroids, as evaluated by flow cytometry. 
A

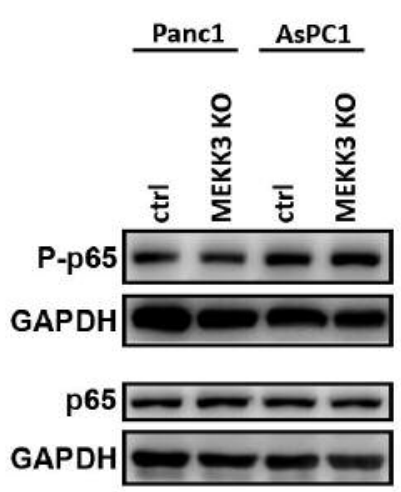

B

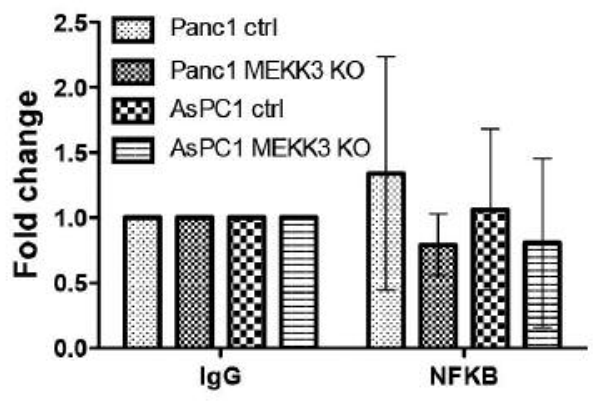

C Birc3

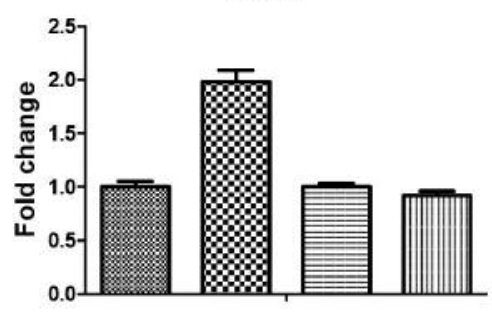

IL1a

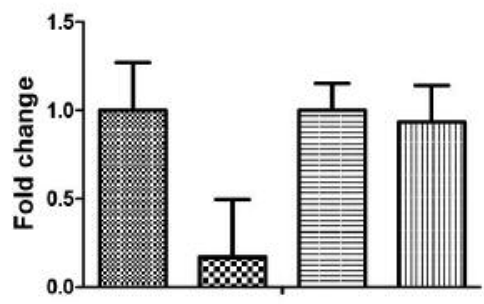

CXCL1

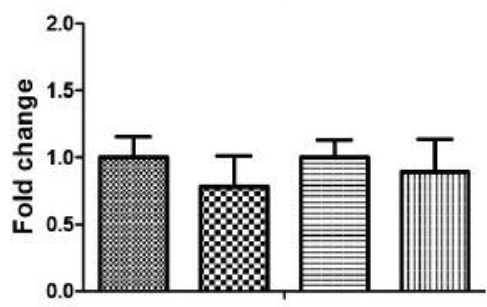

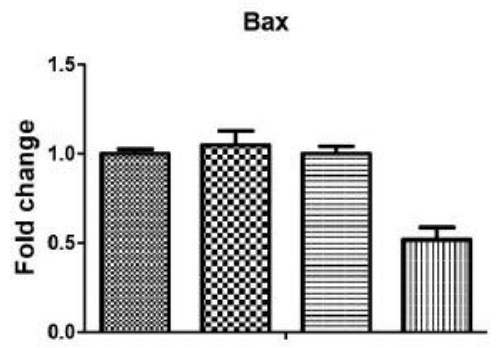

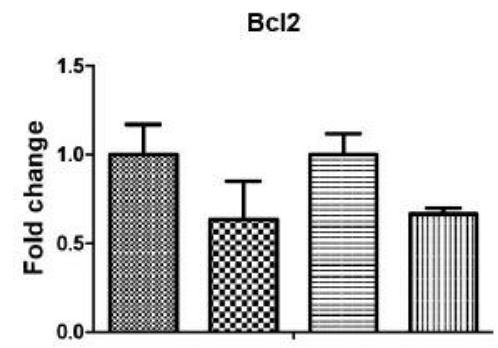

IL1b
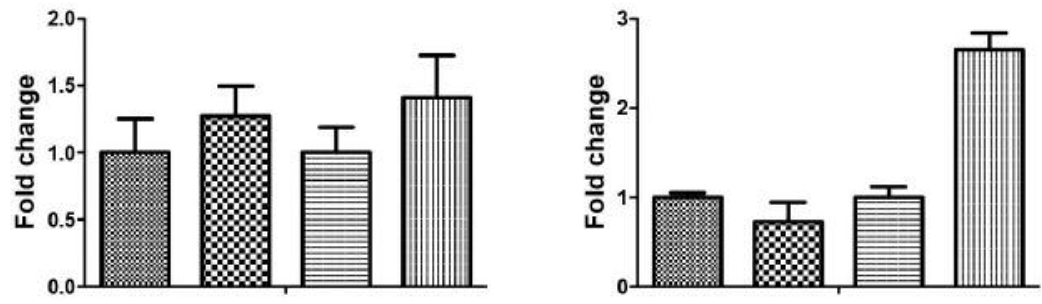

CXCL2
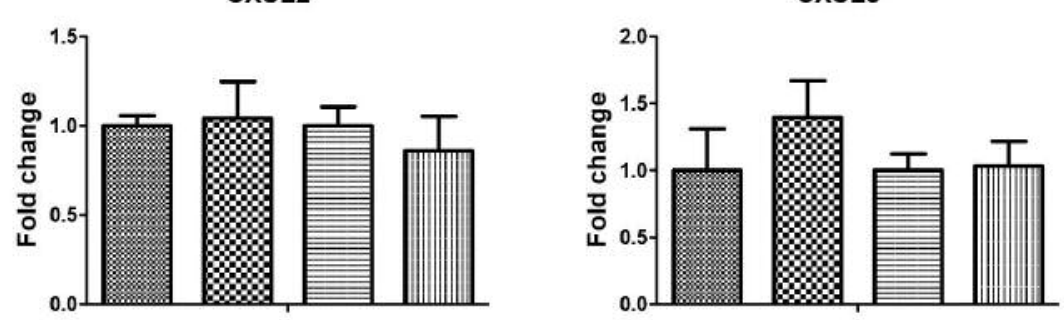

Figure 2. MEKK3 KO does not impair phosphorylation of p65 and does not affect NF-kB target genes expression in pancreatic cancer cell lines. A) Western blot analysis of phosphorylated and total p65 in Panc1, AsPC1 control and MEKK3 KO cells. B) Chromatin immunoprecipitation in control and MEKK3 KO Panc1 and AsPC1 cells. Histograms show fold increase over control (IgG) for Birc3 promoter. Mean values and SD are shown. C) Histograms show mRNA levels of the indicated $N F-k B$ target genes over $\beta$-actin. Mean values and SD are shown.t-test has been used to perform statistical analysis. 

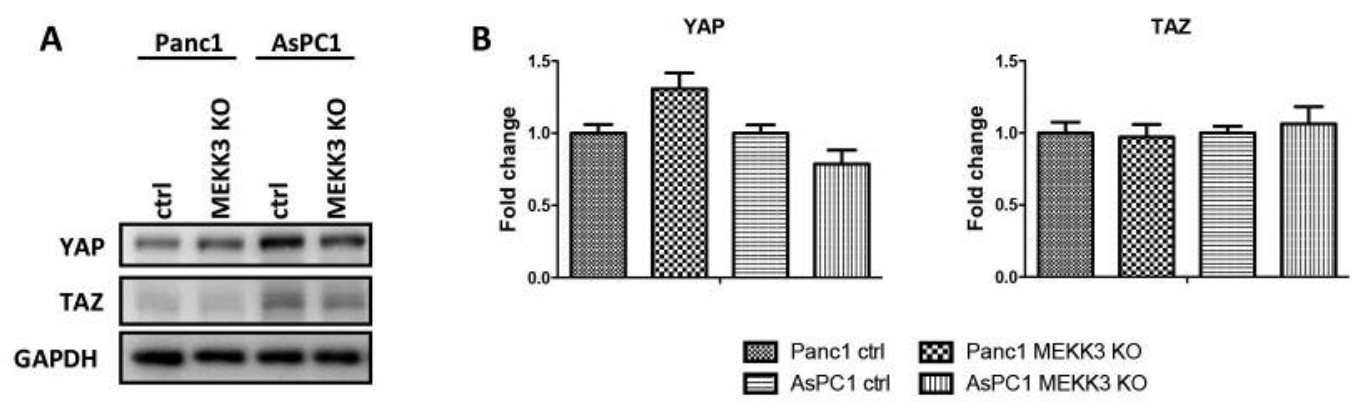

\section{C}
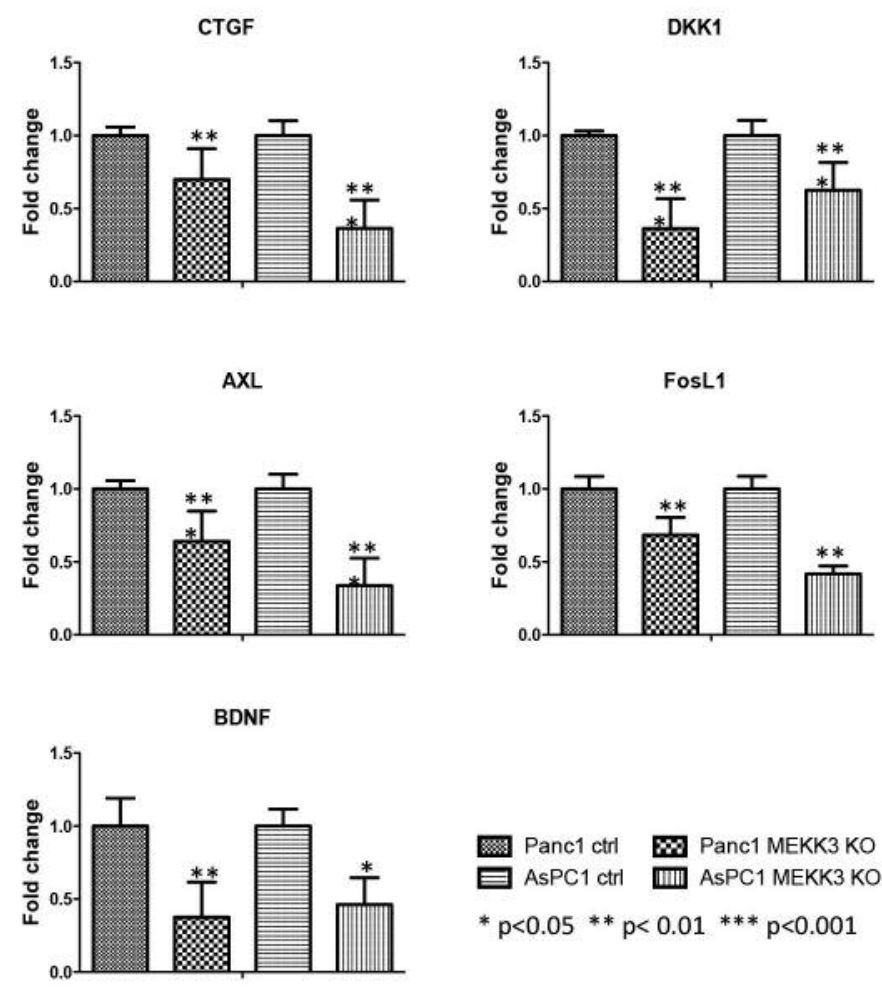

Panc1 ctrl
AsPC1 ctrl
${ }^{*} p<0.05^{* *} p<0.01^{* * *}$ AsPC1 MEKK3 KO

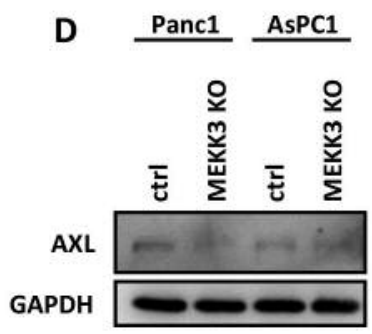

Figure 3. MEKK3 KO reduces the YAP/TAZ target gene expression, but not YAP/TAZ. A) Western blot analysis for the expression of YAP/TAZ in control and MEKK3 KO Pancl and AsPC1 cells. B) Histograms show fold change in mRNA expression of YAP/TAZ over $\beta$-actin by $q R T$ $P C R$. Mean values and SD are shown. t-test has been used to perform statistical analysis. C) Western blot analysis for YAP/TAZ and AXL in the indicated cells. D) Expression levels. Histograms show mRNA levels of the indicated YAP/TAZ target genes over $\beta$-actin. Mean values and $S D$ are shown. $t$-test has been used to perform statistical analysis. $* * * p<0.001 ; * * p<0.01 ; * p<0.05$.

and/or activity of NF-kB. Surprisingly, MEKK3 KO did not modulate the activating phosphorylation of NF-KB (Figure 2A). Accordingly, we could not measure a difference in the recruitment of NF-kB onto its target promoter Birc3 (Figure
2B), or any consistent or significant modulation of the expression of several NF-kB regulated genes (Figure 2C). In conclusion, these data demonstrate that both NF-kB levels and transcriptional activity are not regulated by MEKK3. 

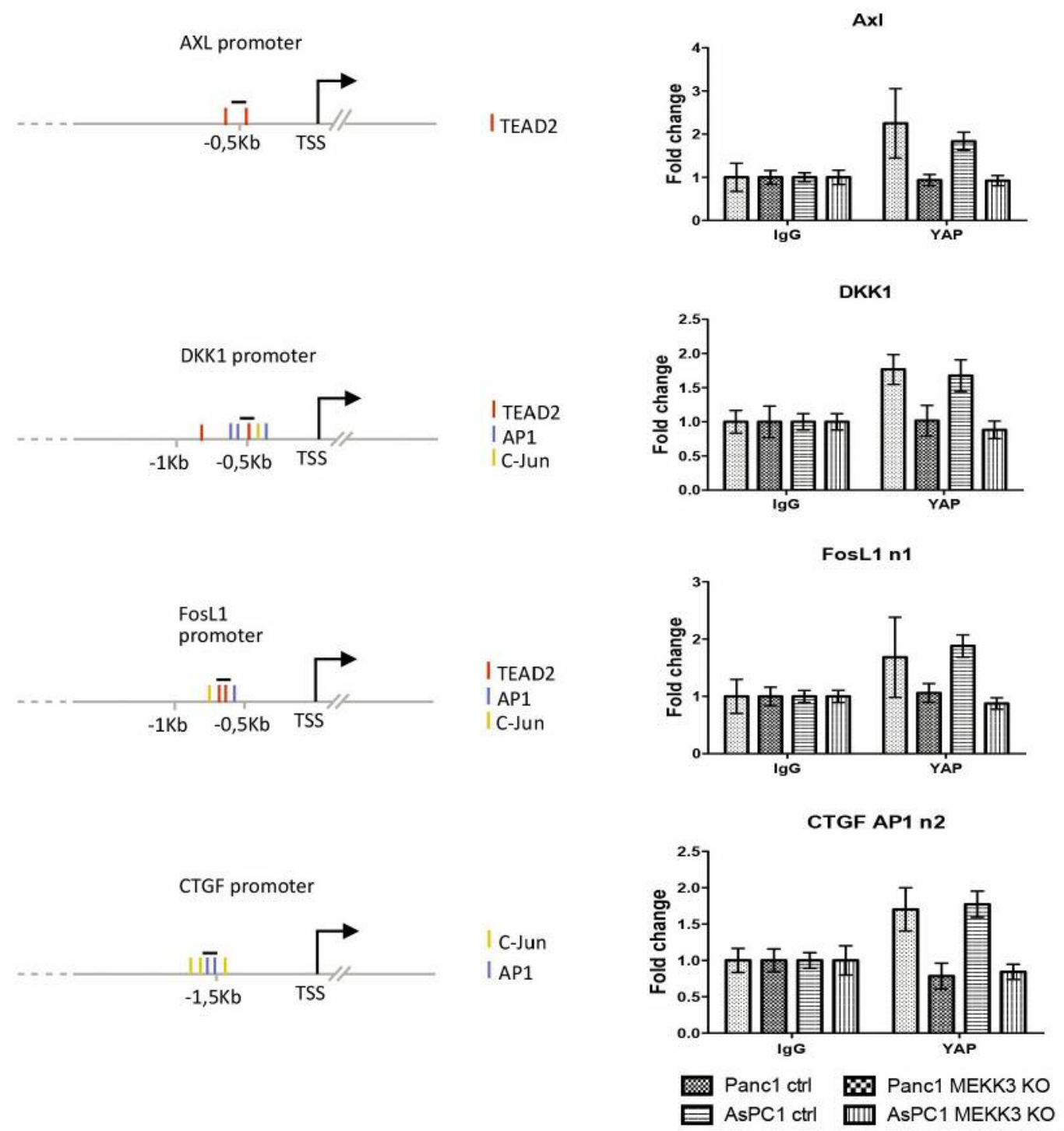

Figure 4. MEKK3 KO reduces the binding of YAP/TAZ to the promoter regions of AXL, DKK1, FosL1 and CTGF. Chromatin immunoprecipitation in control and MEKK3 KO Panc1 and AsPC1. Histograms show fold increase over control (IgG) for AXL, DKK1, FosL1 and CTGF promoters. Mean values and SD are shown. A graphical representation of the promoter regions and transcription factor binding sites is shown on the left.

Because of the growing evidences supporting a role for YAP and TAZ in contributing to pancreatic cancer initiation and progression $(34,35)$, we investigated whether MEKK3 KO could affect YAP/TAZ expression or transcriptional activity. We observed that neither YAP or TAZ proteins nor mRNA levels were significantly affected by MEKK3 KO in Panc1 and AsPC1 cell lines (Figure 3A-B). Nonetheless, when we investigated the transcriptional activity of YAP/TAZ we measured a consistent and significant reduction of the mRNA expression levels of their target genes CTGF, DKK1, AXL, FosL1 and BDNF in MEKK3 $\mathrm{KO}$ cells as compared to their respective controls (Figure 3C). Likewise, we observed a significant reduction of AXL protein levels in MEKK3 KO cells (Figure 3D). Altogether, our results show for the first time an involvement of MEKK3 in sustaining YAP/TAZ transcriptional activity.

These data pointed out the possibility that MEKK3 KO might affect the recruitment of YAP/TAZ to the promoters of their responsive genes. To this end, we measured the capability of YAP/TAZ to immunoprecipitate target regions of chromatin in the promoters of their responsive genes by ChIP assays. Indeed, we observed a complete abolishment of YAP/TAZ binding to the promoter regions of Axl, DKK1 and FosL1 onto TEAD2 binding sites, as well as on AP1 sites in the CTGF promoter (Figure 4). 
A

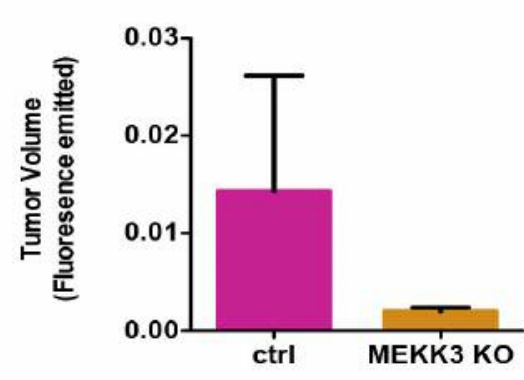

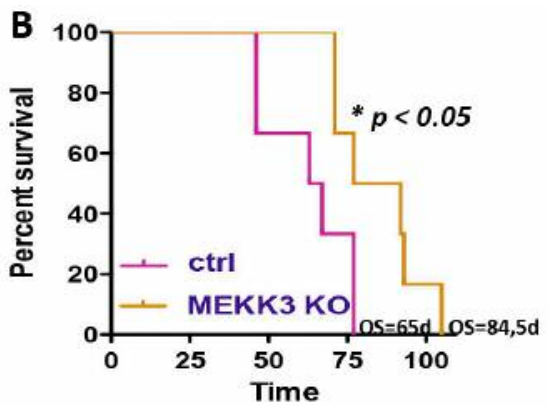

C

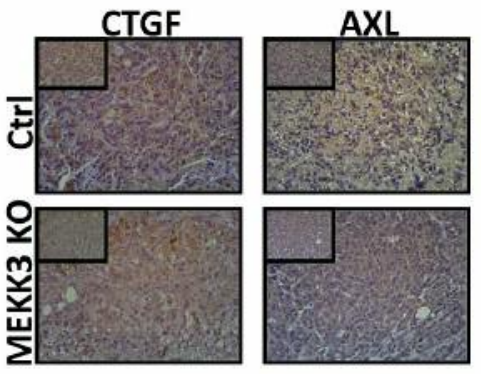

CXCR4

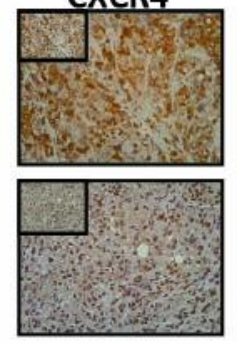

MMP7

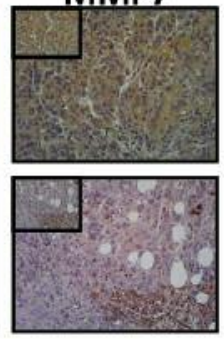

Ki67

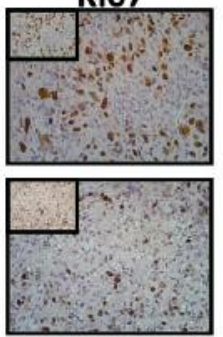

Figure 5. MEKK3 KO affects tumors growth and survival of Panc1 pancreatic tumors. A) Five athymic nude mice were injected with control or MEKK3 KO Pancl cells. Tumor volume was quantified as the average of fluorescence emitted in Pancl within the region of the tumor. Error bars indicate SEM. B) Mice were killed by cervical dislocation when evidence of advanced bulky disease was present. The day of sacrifice was considered the day of death from disease for the purpose of survival evaluation. Differences among survival duration of mice in each group were determined by log-rank test. ${ }^{*} p<0.05$. C) Paraffin-embedded tumor sections stained immunohistochemically with antibodies against Ki67, CTGF, AXL, MMP7 and CXCR4. Images were acquired at 20x magnification; image inserts show 10x magnification of the same section.

Altogether, these data demonstrate that MEKK3 KO inhibits YAP/TAZ oncogenic activities by preventing their recruitment onto their responsive promoters.

MEKK3 KO reduces tumor growth and increases survival of pancreatic cancer orthotopic xenograft models. To determine the in vivo relevance of targeting MEKK3, we evaluated growth and survival of orthotopic control and MEKK3 KO pancreatic cancer xenografts. At the median survival duration of mice in the control group (day 66), mice injected with MEKK3 KO cells experienced a significant reduction of tumor burden (Figure 5A). Accordingly, mice injected with MEKK3 KO cells demonstrated a statistically significant increase in median overall survival (control vs. MEKK3 KO median survival $=66 v s .84 .5$ days, $p=0.0306$ ) (Figure 5B).

Immunohistochemical analyses were carried out to evaluate both the proliferation rate of the tumors and the expression of YAP/TAZ targets contributing to the aggressiveness of pancreatic cancer. We observed a marked reduction of Ki67-positive cells in tumors arisen from MEKK3 KO Panc1 cells (Figure 5C). In addition, the expression of the YAP/TAZ induced proteins CTGF, AXL and MMP7 as well as that of the stemness/migration marker CXCR4 was reduced in MEKK3 KO tumors if compared with controls (Figure 5C).

\section{Discussion}

In this study, we demonstrated that MEKK3 contributes to EMT and stemness in models of pancreatic cancer. This mechanism is independent on the activation of NF-kB and is, however, mediated by the transcriptional coactivators YAP/TAZ.

In recent years, MEKK3 has been involved in the development and the malignancy of different tumors. In particular, MEKK3 induced proliferation, migration and invasion of lung cancer cells, through the activation of AKT and GSK3 $\beta$ signaling pathways (36), metastasis and survival of breast cancer cells (37), as well as chemoresistance of ovarian cancer cells $(38,39)$. MEKK3 has shown clinical relevance in esophageal squamous cell carcinoma, where the combination of its overexpression combined to lymph-node positivity has been shown to be a negative prognostic factor (40).

YAP has been recently demonstrated as a critical oncogenic effector of pancreatic cancer development induced by oncogenic KRAS gene (35). Moreover, two recent papers studied the mechanism of tumor recurrence after KRAS inhibition in pancreatic and lung cancer models, and intriguingly linked the rescue of cell survival of initially KRAS addicted tumor cells to YAP1 activation. In genetically engineered KRAS ${ }^{\mathrm{G} 12 \mathrm{D}:}$ Trp53L/+ mouse models, 
spontaneous relapse of pancreatic cancer developed after KRAS ${ }^{\mathrm{G} 12 \mathrm{D}}$ inactivation relied on YAP1 overexpression as a mechanism for KRAS independent growth $(34,41)$. The Hippo kinase cascade is the main regulator of YAP/TAZ through phosphorylation by the kinase LATS and consequent proteasomal or autophagic degradation. However, recent studies have expanded the complexity of YAP/TAZ identifying new upstream signaling pathways (22). Our study contributes to this field by providing evidences for a role of MEKK3 in sustaining key oncogenic features in vitro and in vivo in pancreatic cancer by inducing the binding of YAP/TAZ to the promoter regions of their regulated genes.

We recently demonstrated that the activation of $\mathrm{NF}-\mathrm{kB}$ mediated by TAK 1 contributes in an essential manner to the chemoresistance of pancreatic cancer (15). Different studies have demonstrated that MEKK3 could affect directly, or indirectly through TAK1, NF-kB activation $(17,20,21)$. Our present results demonstrated that knock-out of MEKK3 did not affect NF-kB in pancreatic cancer, suggesting that TAK1 could be the main inducer of $\mathrm{NF}-\mathrm{kB}$, and in turn of chemoresistance, in this tumor type.

In conclusion, our work identifies MEKK3 as a positive regulator of YAP/TAZ activity in pancreatic cancer, and supports the development of MEKK3 inhibitors which could potentially be used as a mean to target the still undruggable YAP/TAZ transcriptional cofactors in integrated therapeutic strategies (42) in order to improve mortality of this devastating disease.

\section{Conflicts of Interest}

The Authors declare that they have no competing interests.

\section{Acknowledgements}

All Authors read and approved the final manuscript. Animal use was approved by the competent Veterinary Ethics Committee at the University of Verona, and by the Italian Ministry of Health Animal Care and Use Committee.

Part of the work was performed at the Laboratorio Universitario di Ricerca Medica (LURM) Research Center, University of Verona. This work was supported by the Investigator Grant $\mathrm{n}^{\circ} 19111$ to DM and $\mathrm{n}^{\circ} 18599$ to GT through the Associazione Italiana per la Ricerca sul Cancro (AIRC), by the Basic Research Project 2015 through the University of Verona to DM, and by the "Nastro Viola" and "Voglio il Massimo" associations of patients' donations to DM.

\section{References}

1 Melisi D and Budillon A: Pancreatic cancer: between bench and bedside. Current drug targets 13: 729-730, 2012.

2 Melisi D, Calvetti L, Frizziero M and Tortora G: Pancreatic cancer: systemic combination therapies for a heterogeneous disease. Curr Pharm Des 20: 6660-6669, 2014.
3 Vaccaro V, Sperduti I, Vari S, Bria E, Melisi D, Garufi C, Nuzzo C, Scarpa A, Tortora G, Cognetti F, Reni M and Milella M: Metastatic pancreatic cancer: Is there a light at the end of the tunnel? World J Gastroenterol 21: 4788-4801, 2015.

4 Rahib L, Smith BD, Aizenberg R, Rosenzweig AB, Fleshman $\mathrm{JM}$ and Matrisian LM: Projecting cancer incidence and deaths to 2030: the unexpected burden of thyroid, liver, and pancreas cancers in the United States. Cancer Res 74: 2913-2921, 2014.

5 Tamburrino A, Piro G, Carbone C, Tortora G and Melisi D: Mechanisms of resistance to chemotherapeutic and antiangiogenic drugs as novel targets for pancreatic cancer therapy. Front Pharmacol 4: 56, 2013.

6 Vaccaro V, Melisi D, Bria E, Cuppone F, Ciuffreda L, Pino MS, Gelibter A, Tortora G, Cognetti F and Milella M: Emerging pathways and future targets for the molecular therapy of pancreatic cancer. Expert Opin Ther Targets 15: 1183-1196, 2011.

7 Gaianigo N, Melisi D and Carbone C: EMT and treatment resistance in pancreatic cancer. Cancers (Basel) 9, 2017. doi: 10.3390/cancers9090122.

8 Melisi D, Niu J, Chang Z, Xia Q, Peng B, Ishiyama S, Evans DB and Chiao PJ: Secreted interleukin-1alpha induces a metastatic phenotype in pancreatic cancer by sustaining a constitutive activation of nuclear factor-kappaB. Mol Cancer Res 7: 624-633, 2009.

9 Carbone C, Piro G, Simionato F, Ligorio F, Cremolini C, Loupakis F, Ali G, Rossini D, Merz V, Santoro R, Zecchetto C, Zanotto M, Di Nicolantonio F, Bardelli A, Fontanini G, Tortora $\mathrm{G}$ and Melisi D: Homeobox B9 mediates resistance to AntiVEGF therapy in colorectal cancer patients. Clin Cancer Res 23: 4312-4322, 2017.

10 Carbone C, Moccia T, Zhu C, Paradiso G, Budillon A, Chiao PJ, Abbruzzese JL and Melisi D: Anti-VEGF treatment-resistant pancreatic cancers secrete proinflammatory factors that contribute to malignant progression by inducing an EMT cell phenotype. Clin Cancer Res 17: 5822-5832, 2011.

11 Carbone C, Tamburrino A, Piro G, Boschi F, Cataldo I, Zanotto M, Mina MM, Zanini S, Sbarbati A, Scarpa A, Tortora G and Melisi D: Combined inhibition of IL1, CXCR1/2, and TGFbeta signaling pathways modulates in vivo resistance to anti-VEGF treatment. Anti-cancer Drugs 27: 29-40, 2016.

12 Zhuang Z, Ju HQ, Aguilar M, Gocho T, Li H, Iida T, Lee H, Fan X, Zhou H, Ling J, Li Z, Fu J, Wu M, Li M, Melisi D, Iwakura Y, Xu K, Fleming JB and Chiao PJ: IL1 Receptor Antagonist Inhibits Pancreatic Cancer Growth by Abrogating NF-kappaB Activation. Clin Cancer Res 22: 1432-1444, 2016.

13 Carbone C and Melisi D: NF-kappaB as a target for pancreatic cancer therapy. Exp Opin Ther Targets 16(Suppl 2): S1-10, 2012.

14 Melisi D and Chiao PJ: NF-kappa B as a target for cancer therapy. Expert Opin Ther Targets 11: 133-144, 2007.

15 Melisi D, Xia Q, Paradiso G, Ling J, Moccia T, Carbone C, Budillon A, Abbruzzese JL and Chiao PJ: Modulation of pancreatic cancer chemoresistance by inhibition of TAK1. J Nat Cancer Inst 103: 1190-1204, 2011.

16 Santoro R, Carbone C, Piro G, Chiao PJ and Melisi D: TAK-ing aim at chemoresistance: The emerging role of MAP3K7 as a target for cancer therapy. Drug Resist Updat 33-35: 36-42, 2017.

17 Yamazaki K, Gohda J, Kanayama A, Miyamoto Y, Sakurai H, Yamamoto M, Akira S, Hayashi H, Su B and Inoue J: Two mechanistically and temporally distinct NF-kappaB activation pathways in IL-1 signaling. Science Signal 2: ra66, 2009. 
18 Yao J, Kim TW, Qin J, Jiang Z, Qian Y, Xiao H, Lu Y, Qian W, Gulen MF, Sizemore N, DiDonato J, Sato S, Akira S, Su B and Li X: Interleukin-1 (IL-1)-induced TAK1-dependent versus MEKK3-dependent NFkappaB activation pathways bifurcate at IL-1 receptor-associated kinase modification. J Biol Chem 282: 6075-6089, 2007.

19 Avruch J: MAP kinase pathways: the first twenty years. Biochim Biophys Acta 1773: 1150-1160, 2007.

20 Yang J, Lin Y, Guo Z, Cheng J, Huang J, Deng L, Liao W, Chen $\mathrm{Z}$, Liu $\mathrm{Z}$ and $\mathrm{Su} \mathrm{B}$ : The essential role of MEKK3 in TNFinduced NF-kappaB activation. Nat Immunol 2: 620-624, 2001.

21 Huang Q, Yang J, Lin Y, Walker C, Cheng J, Liu ZG and Su B: Differential regulation of interleukin 1 receptor and Toll-like receptor signaling by MEKK3. Nat Immunol 5: 98-103, 2004.

22 Piccolo S, Dupont S and Cordenonsi M: The biology of YAP/TAZ: hippo signaling and beyond. Physiol Rev 94: 1287$1312,2014$.

$23 \mathrm{Yu}$ FX and Guan KL: The Hippo pathway: regulators and regulations. Genes \& development 27: 355-371, 2013.

24 Zanconato F, Forcato M, Battilana G, Azzolin L, Quaranta E, Bodega B, Rosato A, Bicciato S, Cordenonsi M and Piccolo S: Genome-wide association between YAP/TAZ/TEAD and AP-1 at enhancers drives oncogenic growth. Nat Cell Biol 17: 12181227, 2015.

25 Zanconato F, Cordenonsi $\mathrm{M}$ and Piccolo S: YAP/TAZ at the Roots of Cancer. Cancer Cell 29: 783-803, 2016.

26 Piro G, Giacopuzzi S, Bencivenga M, Carbone C, Verlato G, Frizziero M, Zanotto M, Mina MM, Merz V, Santoro R, Zanoni A, De Manzoni G, Tortora G and Melisi D: TAK1-regulated expression of BIRC3 predicts resistance to preoperative chemoradiotherapy in oesophageal adenocarcinoma patients. $\mathrm{Br}$ J Cancer 113: 878-885, 2015.

27 Rosa R, Melisi D, Damiano V, Bianco R, Garofalo S, Gelardi T, Agrawal S, Di Nicolantonio F, Scarpa A, Bardelli A and Tortora G: Toll-like receptor 9 agonist IMO cooperates with cetuximab in K-ras mutant colorectal and pancreatic cancers. Clin Cancer Res 17: 6531-6541, 2011.

28 Mori F, Ferraiuolo M, Santoro R, Sacconi A, Goeman F, Pallocca M, Pulito C, Korita E, Fanciulli M, Muti P, Blandino G and Strano S: Multitargeting activity of miR-24 inhibits long-term melatonin anticancer effects. Oncotarget 7: 20532-20548, 2016.

29 Carbone C, Piro G, Fassan M, Tamburrino A, Mina MM, Zanotto M, Chiao PJ, Bassi C, Scarpa A, Tortora G and Melisi D: An angiopoietin-like protein 2 autocrine signaling promotes EMT during pancreatic ductal carcinogenesis. Oncotarget 6 : 13822-13834, 2015.

30 Carbone C, Piro G, Gaianigo N, Ligorio F, Santoro R, Merz V, Simionato F, Zecchetto C, Falco G, Conti G, Kamga PT, Krampera M, Di Nicolantonio F, De Franceschi L, Scarpa A, Tortora G and Melisi D: Adipocytes sustain pancreatic cancer progression through a non-canonical WNT paracrine network inducing ROR2 nuclear shuttling. Int J Obesity, 2017. doi: 10.1038/ijo.2017.285. [Epub ahead of print]

31 Dalla Pozza E, Dando I, Biondani G, Brandi J, Costanzo C, Zoratti E, Fassan M, Boschi F, Melisi D, Cecconi D, Scupoli MT, Scarpa A and Palmieri M: Pancreatic ductal adenocarcinoma cell lines display a plastic ability to bidirectionally convert into cancer stem cells. Int J Oncol 46: 1099-1108, 2015.

32 Melisi D, Ossovskaya V, Zhu C, Rosa R, Ling J, Dougherty PM, Sherman BM, Abbruzzese JL and Chiao PJ: Oral poly(ADP- ribose) polymerase-1 inhibitor BSI-401 has antitumor activity and synergizes with oxaliplatin against pancreatic cancer, preventing acute neurotoxicity. Clin Cancer Res 15: 6367-6377, 2009.

33 Piro G, Carbone C, Cataldo I, Di Nicolantonio F, Giacopuzzi S, Aprile G, Simionato F, Boschi F, Zanotto M, Mina MM, Santoro R, Merz V, Sbarbati A, de Manzoni G, Scarpa A, Tortora G and Melisi D: An FGFR3 autocrine loop sustains acquired resistance to trastuzumab in gastric cancer patients. Clin Cancer Res 22: 6164-6175, 2016.

34 Kapoor A, Yao W, Ying H, Hua S, Liewen A, Wang Q, Zhong Y, Wu CJ, Sadanandam A, Hu B, Chang Q, Chu GC, Al-Khalil R, Jiang S, Xia H, Fletcher-Sananikone E, Lim C, Horwitz GI, Viale A, Pettazzoni P, Sanchez N, Wang H, Protopopov A, Zhang J, Heffernan T, Johnson RL, Chin L, Wang YA, Draetta $G$ and DePinho RA: Yap1 activation enables bypass of oncogenic Kras addiction in pancreatic cancer. Cell 158: 185197, 2014.

35 Zhang W, Nandakumar N, Shi Y, Manzano M, Smith A, Graham G, Gupta S, Vietsch EE, Laughlin SZ, Wadhwa M, Chetram M, Joshi M, Wang F, Kallakury B, Toretsky J, Wellstein A and Yi C: Downstream of mutant KRAS, the transcription regulator YAP is essential for neoplastic progression to pancreatic ductal adenocarcinoma. Sci Signal 7: ra42, 2014.

36 He Y, Wang L, Liu W, Zhong J, Bai S, Wang Z, Thomas DG, Lin J, Reddy RM, Ramnath N, Carrott PW, Lynch WR, Orringer MB, Chang AC, Beer DG and Chen G: MAP3K3 expression in tumor cells and tumor-infiltrating lymphocytes is correlated with favorable patient survival in lung cancer. Sci Rep 5: 11471, 2015.

37 Fan Y, Ge N, Wang X, Sun W, Mao R, Bu W, Creighton CJ, Zheng P, Vasudevan S, An L, Yang J, Zhao YJ, Zhang H, Li XN, Rao PH, Leung E, Lu YJ, Gray JW, Schiff R, Hilsenbeck SG, Osborne CK, Yang $\mathrm{J}$ and Zhang $\mathrm{H}$ : Amplification and overexpression of MAP3K3 gene in human breast cancer promotes formation and survival of breast cancer cells. J Pathol 232: 75$86,2014$.

38 Samanta AK, Huang HJ, Bast RC Jr. and Liao WS: Overexpression of MEKK3 confers resistance to apoptosis through activation of NFkappaB. J Biol Chem 279: 7576-7583, 2004.

39 Samanta AK, Huang HJ, Le XF, Mao W, Lu KH, Bast RC Jr. and Liao WS: MEKK3 expression correlates with nuclear factor kappa B activity and with expression of antiapoptotic genes in serous ovarian carcinoma. Cancer 115: 3897-3908, 2009.

40 Hasan R, Sharma R, Saraya A, Chattopadhyay TK, DattaGupta S, Walfish PG, Chauhan SS and Ralhan R: Mitogen activated protein kinase kinase kinase 3 (MAP3K3/MEKK3) overexpression is an early event in esophageal tumorigenesis and is a predictor of poor disease prognosis. BMC Cancer 14: 2, 2014.

41 Shao DD, Xue W, Krall EB, Bhutkar A, Piccioni F, Wang X, Schinzel AC, Sood S, Rosenbluh J, Kim JW, Zwang Y, Roberts TM, Root DE, Jacks T and Hahn WC: KRAS and YAP1 converge to regulate EMT and tumor survival. Cell 158: 171184, 2014.

42 Melisi D, Piro G, Tamburrino A, Carbone C and Tortora G: Rationale and clinical use of multitargeting anticancer agents. Curr Opin Pharmacol 13: 536-542, 2013.

Received February 10, 2018

Revised February 22, 2018

Accepted February 26, 2018 